\title{
A pilot study: Women's engagement with a nutrition, lifestyle and health website during pregnancy
}

\author{
R.A.K. Kennedy ${ }^{1}$, L. Mullaney ${ }^{1}$, S. Cawley ${ }^{1}$, N. Daly ${ }^{2}$, M.J. Turner ${ }^{2}$ and D. McCartney ${ }^{1}$ \\ ${ }^{1}$ School of Biological Sciences and Environmental Sustainability and Health Institute (ESHI), Dublin Institute of \\ Technology, Dublin 8, Republic of Ireland and ${ }^{2} U C D$ Centre for Human Reproduction, Coombe Women and Infants \\ University Hospital, Dublin 8, Republic of Ireland
}

Technology supported healthcare interventions are a cost effective, widely accessible means of disseminating health information ${ }^{(1)}$. A recent systematic review found technology to be a potentially safe way of delivering nutrition and lifestyle advice to women in pregnancy; however, further research is warranted to elucidate the degree to which obstetric populations engage with such technology ${ }^{(2)}$. This study aimed to determine the engagement of women with an evidence-based online nutrition and lifestyle website during pregnancy.

Women were recruited at their convenience after sonographic confirmation of a singleton ongoing pregnancy and enrolled in a pilot, single-blinded randomised controlled trial. Women were randomly allocated to either the control group which received standard care, or to the intervention group which received access to a nutrition, lifestyle and health website in addition to standard care. Women's demographic details were recorded in addition to their weight and height. BMI was calculated. Engagement and user interaction with the intervention website between November 2015 and March 2016 was assessed. Webpages were categorised into four categories: pregnancy specific nutrition advice, recipes, pregnancy lifestyle advice and pregnancy symptoms advice. The study received ethical approval from the research ethics committees of Dublin Institute of Technology and the Coombe Women and Infants University Hospital.

The mean age of the women recruited to the intervention group $(n=54)$, was $30 \cdot 7$ years $(18-42$ years) and $59 \cdot 3 \%(n=32)$ were nulliparous. The mean BMI was $24.9 \mathrm{~kg} / \mathrm{m}^{2}$ with $13 \%(n=7)$ of the women classified as obese, $20.4 \%(n=11)$ overweight, $50 \%$ $(n=27)$ ideal weight and $1.9 \%(n=1)$ underweight. Eighty-percent $(n=40)$ of the women made a second visit to the website after their initial login at registration, while five of these women $(9.3 \%)$ made a third and fourth site visit. Women visited between $1-$ 12 individual webpages per site visit and averaged 3.7 individual webpage views per site visit. The table outlines women's preferences for the different areas of the intervention website.

\begin{tabular}{lcc}
\hline Webpages visited & $\begin{array}{c}\text { Number of } \\
\text { views }\end{array}$ & $\begin{array}{c}\text { Average length of stay } \\
\text { (mins) }\end{array}$ \\
\hline Pregnancy specific nutrition advice & 32 & 13.68 \\
Recipes & 26 & 9.78 \\
Lifestyle advice & 9 & $6 \cdot 21$ \\
Pregnancy symptoms advice & 6 & 3.41 \\
\hline
\end{tabular}

This study found that women predominantly engaged with pregnancy specific nutrition advice in an online nutrition, lifestyle and health website during pregnancy. This was followed by recipes, lifestyle advice, and pregnancy symptoms advice. This demonstrates that a nutrition, lifestyle and health website could be employed to communicate evidence-based nutrition advice during pregnancy; however, further research is required to assess the efficacy of this method in relation to clinical outcomes.

1. O’Higgins A, Murphy OC, Egan A, Mullaney L et al. (2014) Ir Med J 107, 313-5.

2. O'Brien OA, McCarthy M, Gibney ER et al. (2014) Eur J Clin Nutr 68, 760-6. 